Full length article

\title{
On the chevron morphology of surface martensite
}

\author{
Annick P. Baur*, Cyril Cayron, Roland E. Logé \\ Laboratory of Thermomechanical Metallurgy, PX Group Chair, Ecole Polytechnique Fédérale de Lausanne, 2002, Neuchâtel, Switzerland
}

\section{A R T I C L E I N F O}

\section{Article history:}

Received 21 March 2019

Received in revised form

16 July 2019

Accepted 13 August 2019

Available online 22 August 2019

\section{Keywords:}

Surface martensite

Variant selection

Chevron morphology

\begin{abstract}
A B S T R A C T
It is established that the annealing twin boundaries of austenite act as nucleation sites for the martensitic transformation. In the present study, we observe that in surface martensite the transformation only takes place for twin boundaries that are approximately vertical in respect with the sample free surface. This phenomenon is shown to be related to variant selection. The criterion for surface martensite formation introduced in a previous study is used here to account for the selection of the twin boundaries that promote the transformation. This criterion correctly captures the phenomenon and explains the highly symmetrical chevron morphology observed in surface martensite.
\end{abstract}

(c) 2019 Acta Materialia Inc. Published by Elsevier Ltd. All rights reserved.

\section{Introduction}

The formation of surface martensite in steels was reported several times in the past and studied in details by Klosterman and Burgers [1]. Different alloys (stainless steels, iron-nickel and ironnickel-mangenese alloys) exhibit this particular form of martensite [2-4]. Recently, the present authors showed that a natural variant selection takes place in the martensite formed at the surface of Fe-30 wt\%Ni alloys [5]. The phenomenon is caused by the presence of the free surface which favours the nucleation of some particular martensite orientations. The selection was modeled by using a criterion for martensite formation based on the maximisation of the extension of a material fibre oriented along the normal to the free surface. Different transformation models were compared and we showed that the FCC-BCC distortion associated with the Kurdjumov-Sachs orientation relationship [6,7] convincingly accounts for both the variant selection and the crystallographic features of surface martensite, such as the $\{225\}_{\gamma}$ habit plane and the Kurdjumov-Sachs orientation relationship. Like free surfaces, grain boundaries are also known to promote the nucleation of martensite [8]. Experiments on annealed samples show that twin boundaries in particular act as potent nucleation site for the martensitic transformation, in both surface and bulk martensite [2,9-11]. In the present study, the formation of surface martensite is studied in a cold-worked and recrystallized Fe-30 wt\%Ni alloy,

\footnotetext{
* Corresponding author.

E-mail address: annick.baur@epfl.ch (A.P. Baur).
}

exhibiting a high number of annealing twin boundaries. We observe that not all the twin boundaries are equivalently efficient to promote the formation of surface martensite. Only the boundaries with particular spatial orientations allow the nucleation of martensite. The phenomenon is studied by considering the criterion for surface martensite formation introduced in our previous study [5]. We show that this criterion explains the austenite spatial orientations for which the transformation preferentially takes place, which rationnalizes the highly symmetrical morphology observed in the surface martensite.

\section{Experimental method}

Fe-30 wt\%Ni samples have been cold-worked and recrystallized in quartz tubes at $1100{ }^{\circ} \mathrm{C}$ for $3 \mathrm{~h}$. They were grinded and polished manually with a final stage using a diamond suspension of $1 \mu \mathrm{m}$. The manually polished samples have then been electropolished with Struers A2 electrolyte at $35 \mathrm{~V}$ for 20 s. The temperature of the electrolyte was about $10^{\circ} \mathrm{C}$. It is above the Ms temperature of $\mathrm{Fe}-$ $30 \mathrm{wt} \% \mathrm{Ni}$ bulk martensite, which is reported to be lower than $0^{\circ} \mathrm{C}$ [12]. Surface martensite appears during electropolishing. This transformation above the Ms is believed to be caused by the removal of the stresses on the polished surface. Such transformation is helped by the relatively big austenite grain size (few hundreds microns) obtained after the heat treatement. The produced martensite is characterized by using an XLF-30 (FEI) scanning electron microscope, equipped with a Channel5/Aztec (Oxford Instruments) EBSD system. 


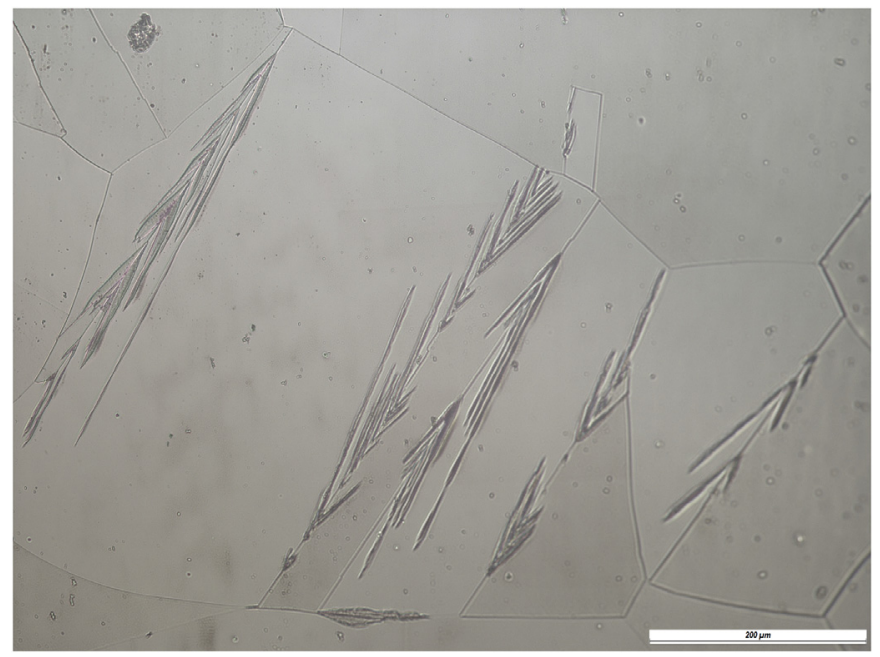

Fig. 1. Optical micrograph of the chevron martensite formed on the surface of the recrystallized Fe-30 wt\% Ni sample.

\section{Results and discussion}

\subsection{Crystallographic characterization}

By analyzing the entire polished surface, we observe that almost all the martensite products have a highly symmetrical chevron morphology. This morphology is illustrated in the optical micrograph in Fig. 1 and in the EBSD measurements of Fig. $2 \mathbf{A}$ and $4 \mathbf{A}$.

The measurement in Fig. 2 indicates that the two austenite grains (yellow and green) are misoriented by a $60^{\circ}$ rotation about $[111]_{\gamma}$. The trace of the grain boundary is perpendicular to the common $[111]_{\gamma}$ and indicated by the circle on Fig. $2 \mathbf{B}$. The corresponding grain boundary is thus an annealing twin boundary. The nucleation of surface martensite exhibiting the chevron morphology appears to be related to the presence of such boundaries. Fig. 3 illustrates schematically the morphology and the crystallography of chevron martensite. Fig. $3 \mathbf{A}$ shows two twinrelated austenite grains ( 1 and 2 ) separated by a twin boundary in red. On each side of the twin boundary, a lenticle of martensite appears. The chevron consists in the assembly of these two lenticles. A detailed crystallographical analysis based on the EBSD measurement suggests that the habit plane of each lenticle is of

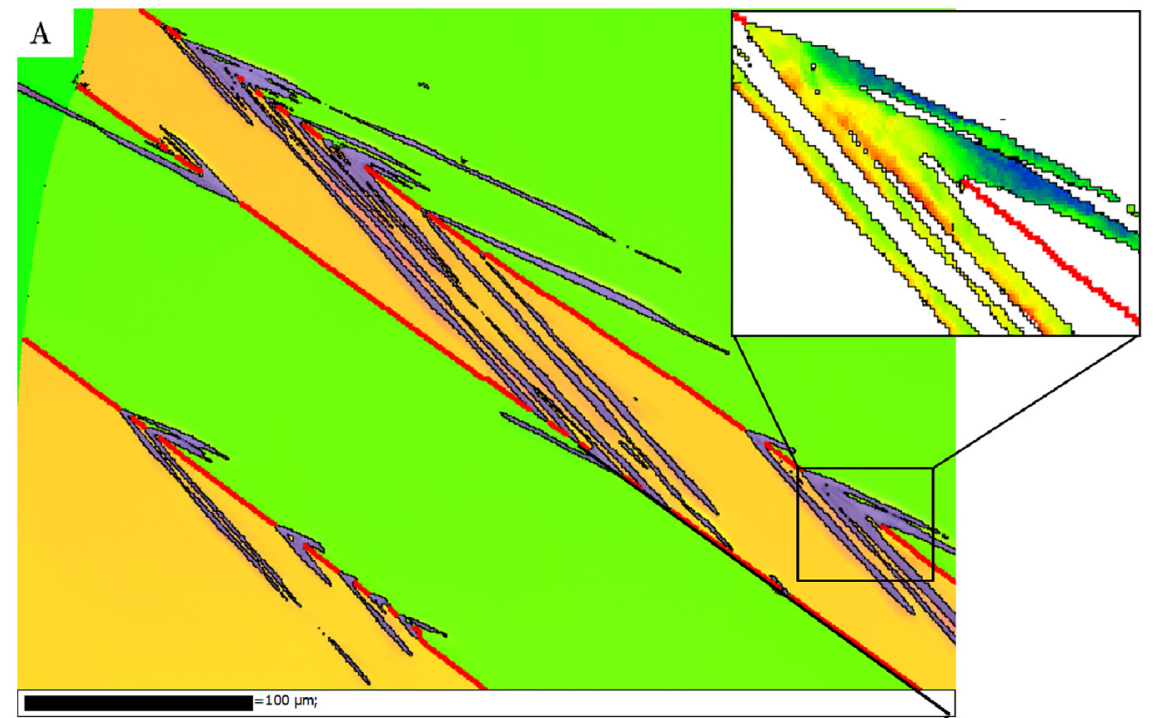

B
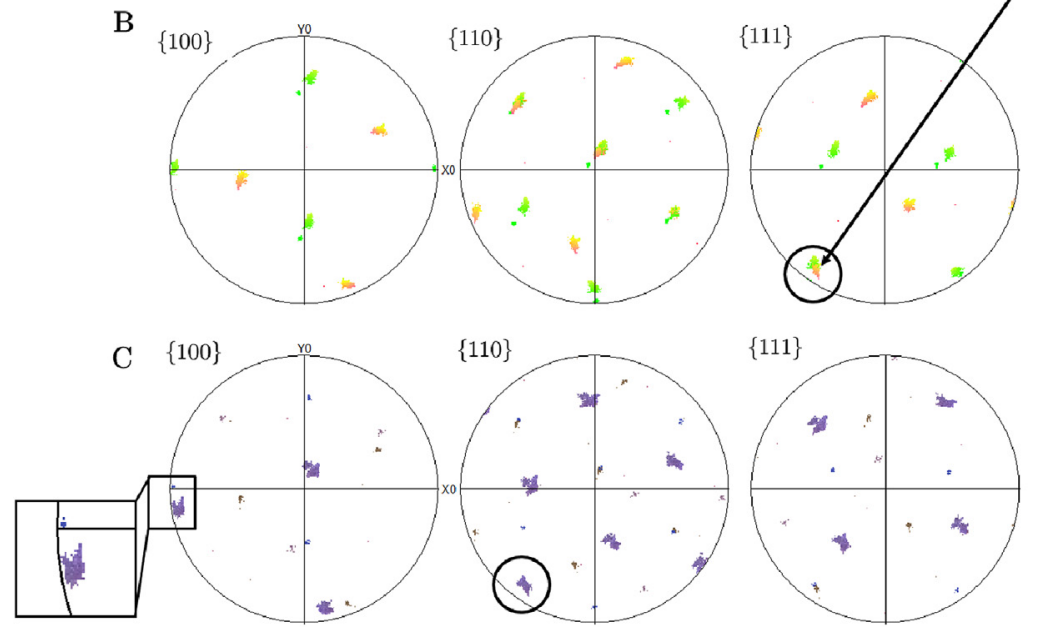

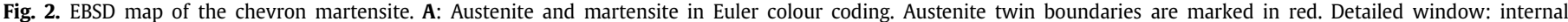

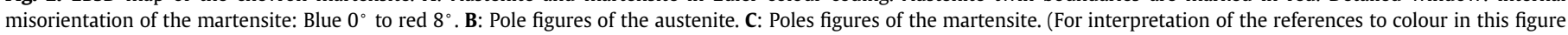
legend, the reader is referred to the Web version of this article.) 
A

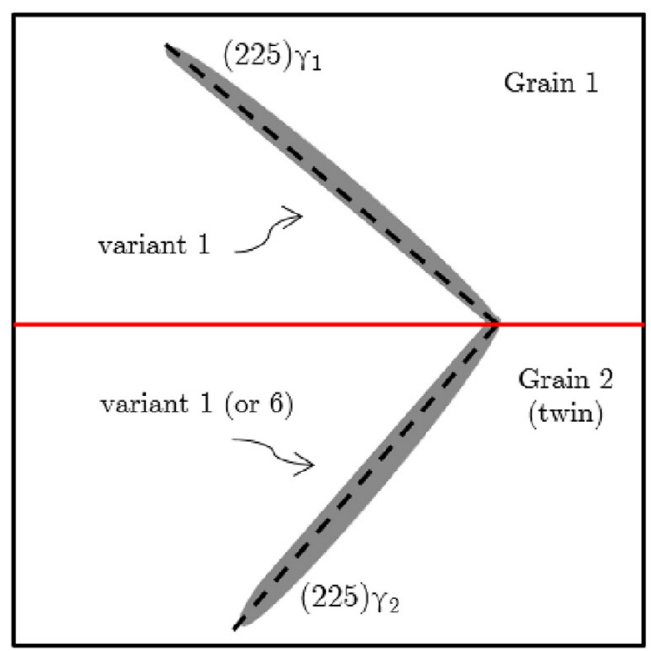

B

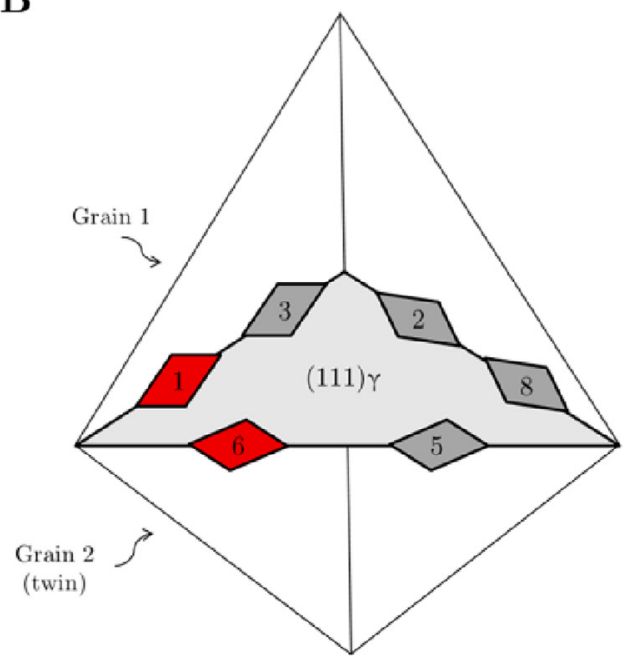

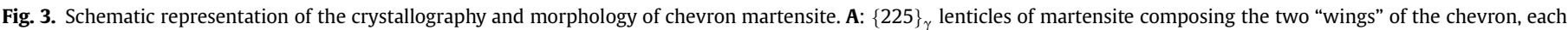

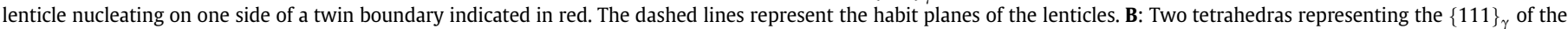

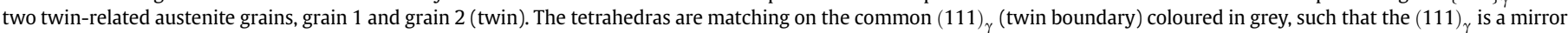

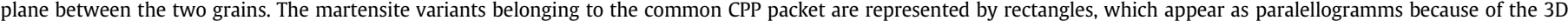

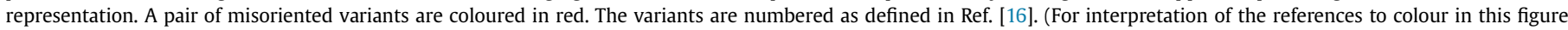
legend, the reader is referred to the Web version of this article.)

type $\{225\}_{\gamma}$, and the orientation relationship is close to Kurdjumov-Sachs, in agreement with our previous study of surface martensite (see supplement B for the details) [5]. Fig. 3B shows two twin-related grains ( 1 and 2 ) represented with the $\{111\}_{\gamma}$ planes. The common $(111)_{\gamma}$, which is the twin boundary, is marked in grey. The six Kurdjumov-Sachs variants having their close-packed plane parallel to the common $(111)_{\gamma}$ close-packed plane are represented with paralellograms. These six variants belong to the same closepacked plane packet (CPP packet). When the close-packed plane associated with these variants corresponds to the twin boundary, the variants have the particularity of being common to both twinrelated grains. From the EBSD measurements in Fig. 2, the variants forming the chevron correspond to that case. The common $(1 \overline{10})_{\alpha} \|(111)_{\gamma}$ is indicated in Fig. 2 with circles on the $\{110\}_{\alpha}$ and $\{111\}_{\gamma}$ poles figures (Fig. $2 \mathbf{B}$ and $\mathbf{C}$ ). As a consequence, the chevron is in Kurdjumov-Sachs orientation relationship with both grains. The propensity of martensite to nucleate in orientation relationship with two different neighbour grains is well documented. This particularity is, for exemple, used as variant selection criterion by Tomida et al. [13] and its implication on the micro-mechanics of steel has been recently enlighted by Archie et al. [14]. The same nucleation behaviour is also observed in the heterogeneous nulceation of BCC precipitates formed on FCC annealing twin boundaries in $\mathrm{Ni}-\mathrm{Cr}$ alloys and in the nucleation of FCC precipitates formed on the BCC deformation twin boundary in a duplex stainless steel [15].

The EBSD characterization of the martensite chevron (Fig. 2C) further indicates that the martensite orientation, in purple, spreads between the two low-misoriented Kurdjumov-Sachs variants belonging to the same CPP packet. The inserted window in Fig. $2 \mathbf{A}$ shows the misorientation inside the chevron. The reference orientation is taken on the top right edge of the chevron, in blue. The gradient of colour indicates the gradient of misorientation from $0^{\circ}$ (blue) to $8^{\circ}$ (red). We measure about $3^{\circ}$ to $5^{\circ}$ of misorientation between the two sides of the chevron. Less than $2^{\circ}$ of misorientation is measured inside each wing of the chevron, this misorientation being probably due to the accommodation of the martensite in the austenite. Exemple of such pair of low- misoriented variants are illustrated in red in Fig. 3B. Among the six variants of the CPP that are in orientation relationship with both twin-related grains, a maximum of two low-misoriented variants appear. In fact, as schematically illustrated in Fig. $3 \mathbf{A}$, the lenticles composing the chevron are either made of the same variant (1), or of the two low-misoriented variants $\left(1\right.$ and $\left.6^{1}\right)$. This suggests that a variant selection takes place inside the CPP packet. The phenomenon was expected as it has already been evidenced and modeled in our recent study of surface martensite [5].

As can be observed on the low magnification EBSD maps in Fig. $4 \mathbf{A}$, the reproducibility of the morphology produced in our sample is striking; the chevron morphology is very frequently observed (about $90 \%$ of all cases). Rarely, the martensite is seen to form on one side of the twin boundary only. We called this unfrequent morphology of martensite half-chevron. Maps showing halfchevrons are presented in supplement $A$. Another exception of the chevron morphology is illustrated in Fig. $4 \mathbf{A}$ and indicated by a dashed arrow on the lower left corner. Some martensite with no particular morphology appears on a grain boundary, which is not an annealing twin. The latter nucleation has been observed in other maps, but the amount of such allotriomorph martensite is always small. It is worth mentionning that the chevron martensite is different from the butterfly martensite, as the chevrons are the result of the martensite nucleation in two different grains, contrarily to the butterfly martensite which forms in a single austenite grain [17-19]. The symmetry of the chevron suggests that the twin boundary where the martensite forms has a particular orientation with respect to the sample free surface. If one considers that the martensite orientation on both sides of the twin boundary is the same, the perfect symmetry is reached only for vertical orientation of the twinning plane, namely when the normal to the twinning plane is at $90^{\circ}$ from the surface normal. To verify experimentally this hypothesis, the angle between the normal to the twinning plane and the surface normal is measured in different

\footnotetext{
${ }^{1}$ We mention here that the variant numbering proposed in this study is the same as defined in Ref. [16].
} 
grains using EBSD measurements. As an example of this analysis, the measured angles are indicated in Fig. 4A. We annotated in black the twin boundaries where martensite is observed and in red where no martensite appears. Various EBSD maps were acquired to increase the statistics. An histogram summarizing these measurements is presented in Fig. $4 \mathbf{B}$. We marked in black the twin boundaries where no martensite forms, in purple the occurrences where half-chevrons are observed, and in green the cases where full chevrons are formed. The maps associated with the measurements shown in Fig. 4B are presented in supplement A. For geometrical reasons, twin boundaries with high angles are more often seen when examining a sample surface than those with low angles. For example, a twin boundary parallel to the surface (angle of $0^{\circ}$ ) cannot be observed by surface analysis. This consideration explains why the histogram shows no twin boundaries with angles lower than $20^{\circ}$. The histogram indicates that there are two thresholds for the orientation of the twin boundaries to promote the nucleation of martensite. The angle between the normal to the twin boundaries where martensite nucleates and the free surface normal is greater than $44^{\circ}$. Half-chevrons are observed for angles between $44^{\circ}$ and $55^{\circ}$, and above this value with a single exception at $68^{\circ}$, the morphology is always the classical chevron. The mean angle where chevron martensite is observed is $77^{\circ}$, while the mean angle associated with all observed twin boundaries is $65^{\circ}$. The measurements thus suggest that there is a selection of the twin boundaries where surface martensite forms, the relatively vertical twin boundaries being more efficient nucleation sites than randomly oriented ones. From our experimental measurements, we conclude that the nucleation of martensite is affected by different factors. First the nucleation takes place on the twin boundary and the martensite is in Kurdjumov-Sachs orientation relationship with both twin-related parent crystals. This implies that the variants of martensite that form belong to the CPP packet associated with the twin boundary. Second, among the six variants of the CPP, a maximum of two variants are observed per twin boundary, these variants being low-misoriented. Finally, it also appears that not all the twin boundary are equivalently efficient in promoting the phase transformation, the chevrons forming preferentially on rather vertical twin boundaries.

\subsection{Variant selection modelling}

We propose to address the selection phenomenon occurring inside the CPP packet, by using our model for variant selection in

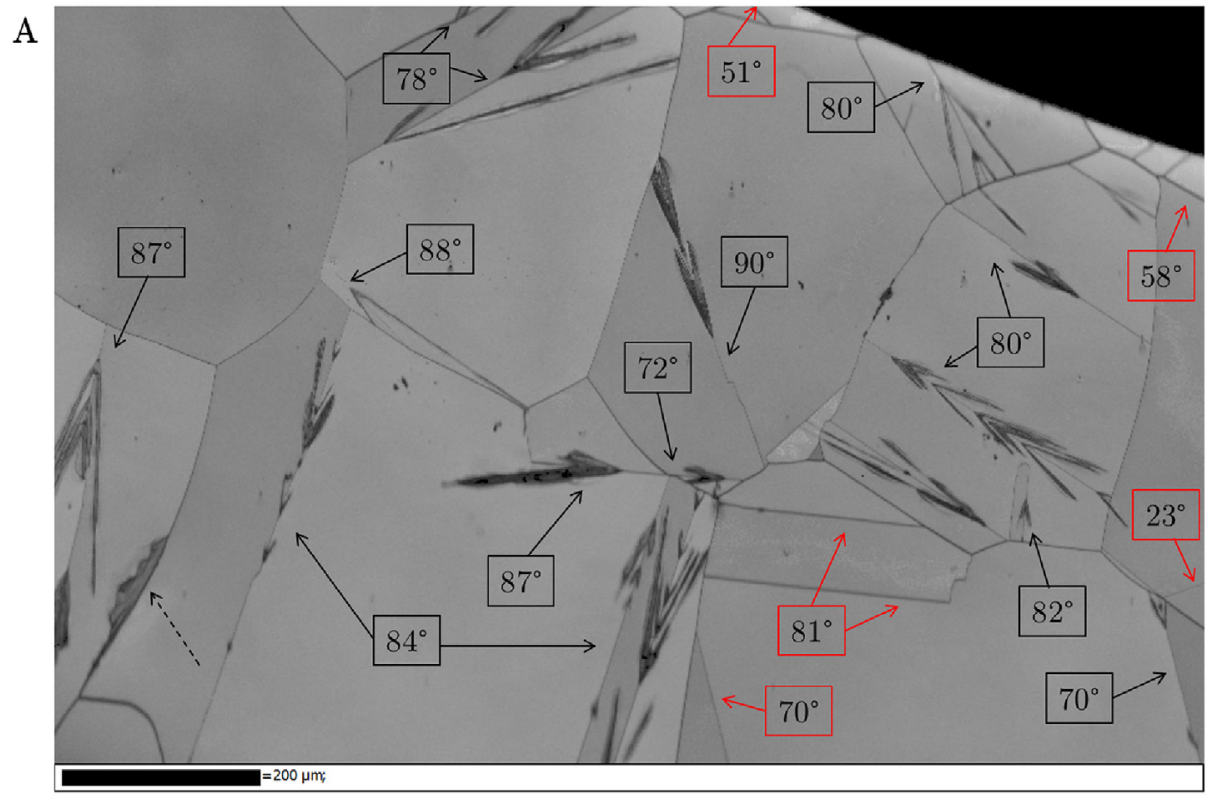

B

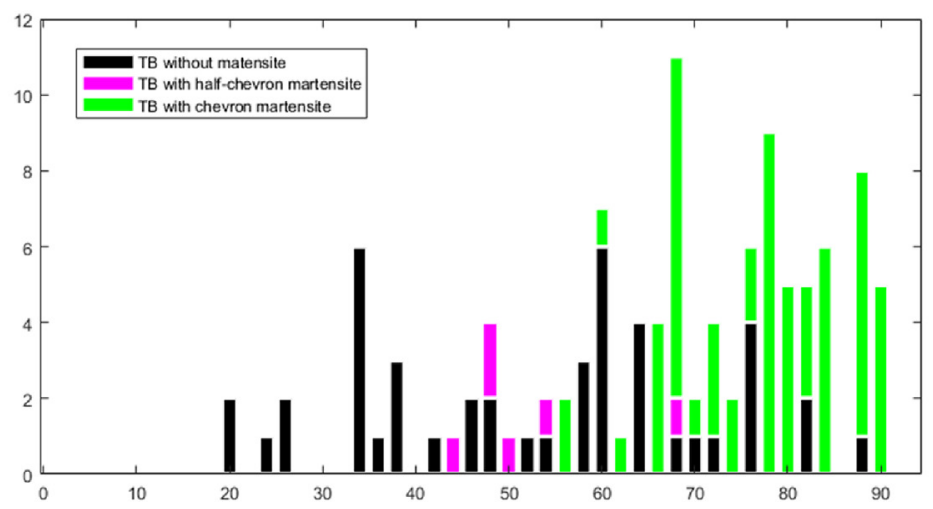

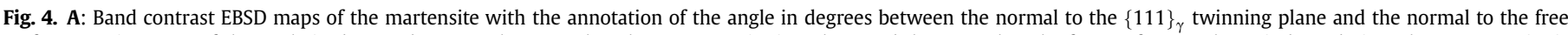

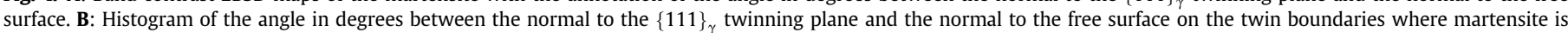

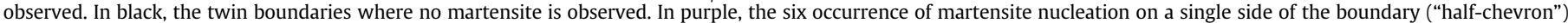

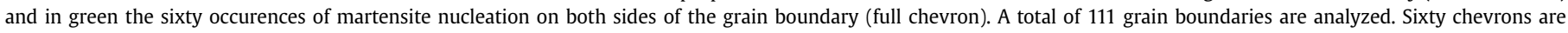
observed and six half-chevrons. (For interpretation of the references to colour in this figure legend, the reader is referred to the Web version of this article.) 
surface martensite presented in Ref. [5]. We define the favoured variants as those that maximize the $z z$ component of the gradient of displacement $\mathbf{H}=\left.\mathbf{F}\right|_{x=\frac{1}{3}}-\mathbf{I}$, when expressed in the sample basis: $\operatorname{Max}\left(\mathrm{H}_{z z}\right)$. The sample basis is such that the $z$-direction is normal to the free surface. This criterion is computed using the continuous FCC-BCC distortion $\mathbf{F}(x)$ where $x$ varies from $x=\frac{1}{2}$, no transformation, to $x=\frac{1}{3}$ describing the complete transformation [7]. The complete transformation corresponds to the Jaswon and Wheeler model with FCC and BCC lattice parameters in hard sphere ratio [6]. The complete distortion is expressed as follows:

$\mathbf{F}\left(\frac{1}{3}\right)=\left[\begin{array}{ccc}\frac{\sqrt{6}}{18}+1 & \frac{\sqrt{6}}{18} & -\frac{\sqrt{6}}{6}+\frac{1}{3} \\ \frac{\sqrt{6}}{18}-\frac{1}{3} & \frac{\sqrt{6}}{18}+\frac{2}{3} & -\frac{\sqrt{6}}{6}+\frac{1}{3} \\ -\frac{\sqrt{6}}{9}+\frac{1}{3} & -\frac{\sqrt{6}}{9}+\frac{1}{3} & \frac{\sqrt{6}}{3}+\frac{1}{3}\end{array}\right]$

The continuous model is chosen to model the transformation as it better accounts for the Kurdjumov-Sachs orientation relationship and the $\{225\}_{\gamma}$ habit plane than the PTMC (Phenomenolocial Theory of the Martensite Crystallography) predictions using the Fe$30 \mathrm{wt} \% \mathrm{Ni}$ lattice parameters [5]. As compared to our original model in Ref. [5], we add here an additional hypothesis on the nucleation of martensite. We consider that martensite nucleates only on the twin boundaries and that it is in Kurdjumov-Sachs orientation relationship with both grains. We thus restrict the number of variants that could form to the six variants belonging to the CPP associated with the twin boundary. From the EBSD measurements we extract the two austenite orientations and for each of them we compute the distortion associated with the six aforementioned variants. In each grain, the variant exhibiting the highest $H_{z z}$ value is selected. The result of the simulation are presented in the pole figures in Fig. 5A and 5B for the green and the yellow grains, respectively.

For each austenite orientation, the 24 Kurdjumov-Sachs variants are represented by empty circles. The variant selected in the green grain is marked in green on both Fig. $5 \mathbf{A}$ and $\mathbf{B}$, similarly the variant selected in the yellow grain is marked in orange, for a better visibility. Superimposed on the simulations, we plotted the experimental martensite orientations of Fig. 2 in black. The simulation indicates that the variant that is selected in the green grain is lowmisoriented with the variant selected in the yellow grain. The simulation and the experimental measurements are in good agreement. The same quality of results is obtained for two additional simulations proposed in supplement $\mathrm{A}$.

Consequently, we propose to apply our criterion for variant selection to explain the selection of the twin boundary on which the martensite preferentially nucleates, and to rationalize its chevron morphology. We explore the space of all possible orientations of an austenite crystal with respect to a free surface normal to $z$, and evaluate $H_{z z}$ for a given variant. The variant considered in the computation (equation (1)) has the invariant $[1 \overline{1} 0]_{\gamma}$ direction and the untilted $(111)_{\gamma}$ plane. This variant belongs to the $(111)_{\gamma}$ CPP packet and accordingly is associated with a (111) ${ }_{\gamma}$ twin boundary. We choose to discretize the space of the possible orientations of austenite using two rotations: the first one around $[1 \overline{1} 0]_{\gamma}$ and the second one around $[111]_{\gamma}$. Because the $H_{z z}$ expresses a projection along the normal to the sample surface, only two rotations are necessary to explore the space.

The angular step of each rotation is $0.01 \mathrm{rad}$. The initial orientation of the austenite crystal is such that the $[1 \overline{1} 0]_{\gamma}$ direction is along the $x$-axis of the sample, the $[11 \overline{2}]_{\gamma}$ along the $y$-axis and the
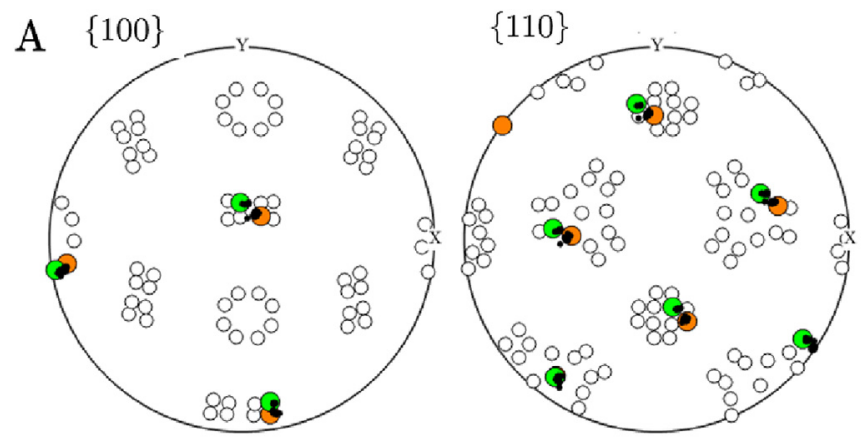

B

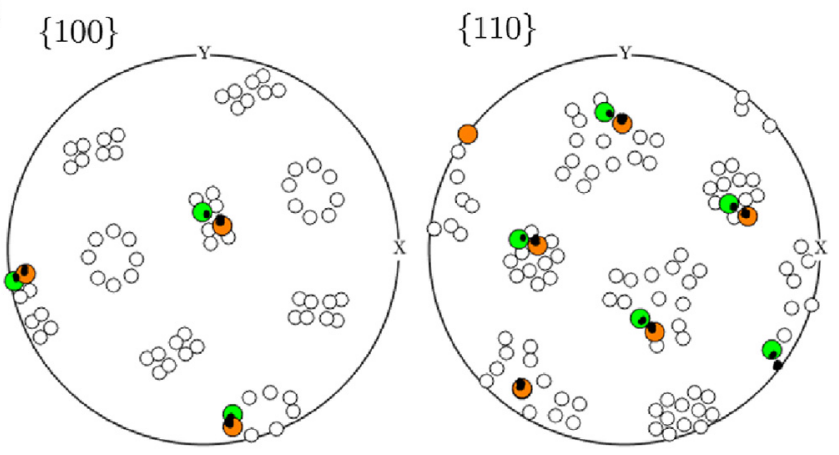

Fig. 5. Simulated pole figure of the chevron martensite in Fig. 2. A: Simulation in the green grain. B: Simulation in the yellow grain. In green, the orientation selected by the maximum work criterion in the green grain and in orange the orientation selected by the maximum work criterion in the yellow grain. The experimental orientations are superimposed in black. (For interpretation of the references to colour in this figure legend, the reader is referred to the Web version of this article.)

$[111]_{\gamma}$ along the $z$-axis, namely along the normal to the free surface. This initial orientation is illustrated in Fig. 6A. Fig. $6 \mathbf{C}$ shows a map of the value of $H_{z z}$ as function of the two rotation angles. The first rotation around $[1 \overline{1} 0]_{\gamma}$ is represented on the $\theta$-axis and the second around $[111]_{\gamma}$ is represented on the $\phi$-axis. In Fig. $6 \mathbf{D}$, we plotted the maximal value of $H_{z z}$ found as function of the $\theta$ angle between the $[111]_{\gamma}$ and the normal to the free surface. The dependence of the $H_{z z}$ value to the angle between the surface normal and [111 $]_{\gamma}$ has a symmetrical shape about $90^{\circ}$. The maximum value is 0.1528 and it corresponds to the angles of $73^{\circ}$ (and $107^{\circ}$ ). This variant is thus favoured when its close-packed plane, hence the twin boundary, is rather vertical with respect to the free surface normal. An illustration of that situation is proposed in Fig. $6 \mathbf{B}$. The experimental measurements reported in Fig. 4D show that there is not a unique orientation of the twinning plane that favours the martensite nucleation. The angle $\theta$ between the normal to the $\{111\}_{\gamma}$ twin boundary where martensite forms, with either a half or a full chevron, and the normal to the sample free surface spreads from about $44^{\circ}$ to $90^{\circ}$. By using a threshold value on the $\mathbf{H}_{z z}$ criterion for the nucleation of martensite, we select a number of austenite orientations that are the most efficient to promote the transformation. This threshold is set to mimik the experimental range. It is found to be about $95 \%$ of the maximum value of the $H_{z z}$ component, namely 0.1452 , such as to reach a threshold angle of $44^{\circ}$. The threshold $H_{z z}$ value and the corresponding angles are illustrated on Fig. $6 \mathbf{C}$ and $\mathbf{D}$ with the dashed red lines. The orientation satisfying the $95 \% \cdot \max \left(H_{z z}\right)$ condition are marked in white in Fig. 6C.

It has been seen before that the martensite transformation tends to take place on both sides of the twin boundary, so we also evaluate the value of $H_{z z}$ on the other side of the twin boundary, namely in grain 2, if one refers to the sketch in Fig. $6 \mathbf{B}$. This computation is 
A

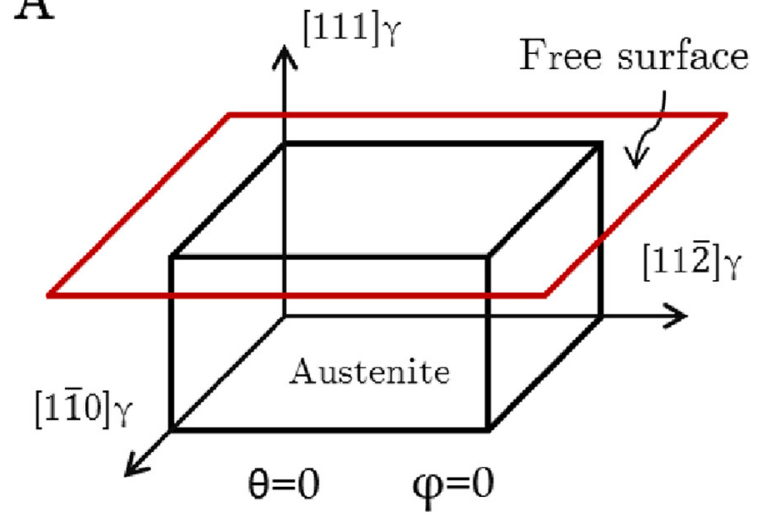

C $44^{\circ}$ $136^{\circ}$

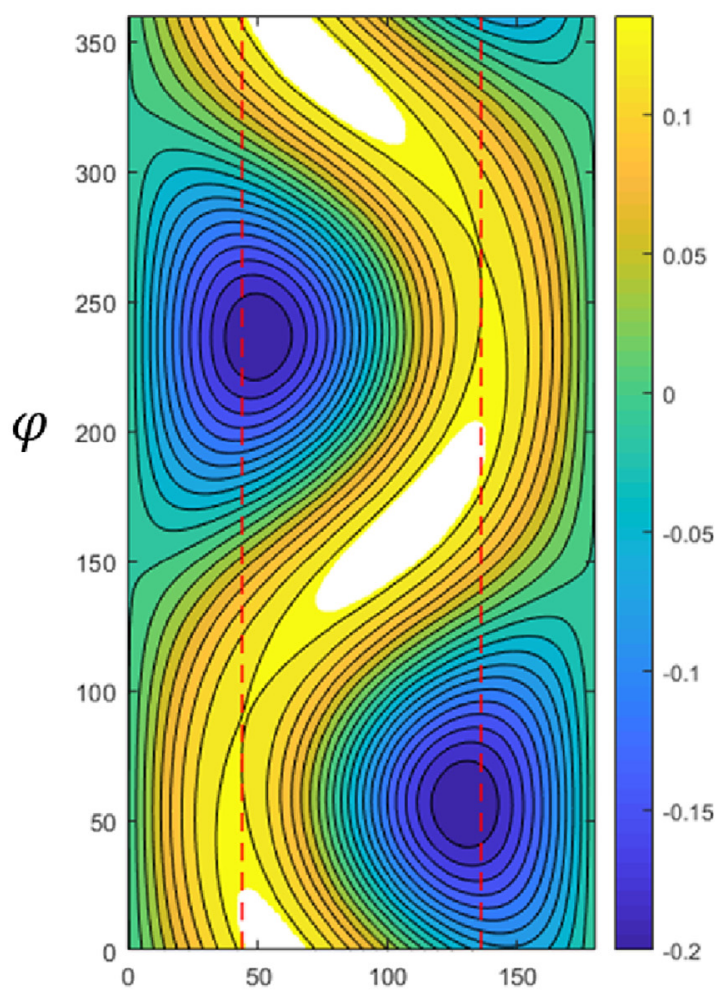

$\theta$

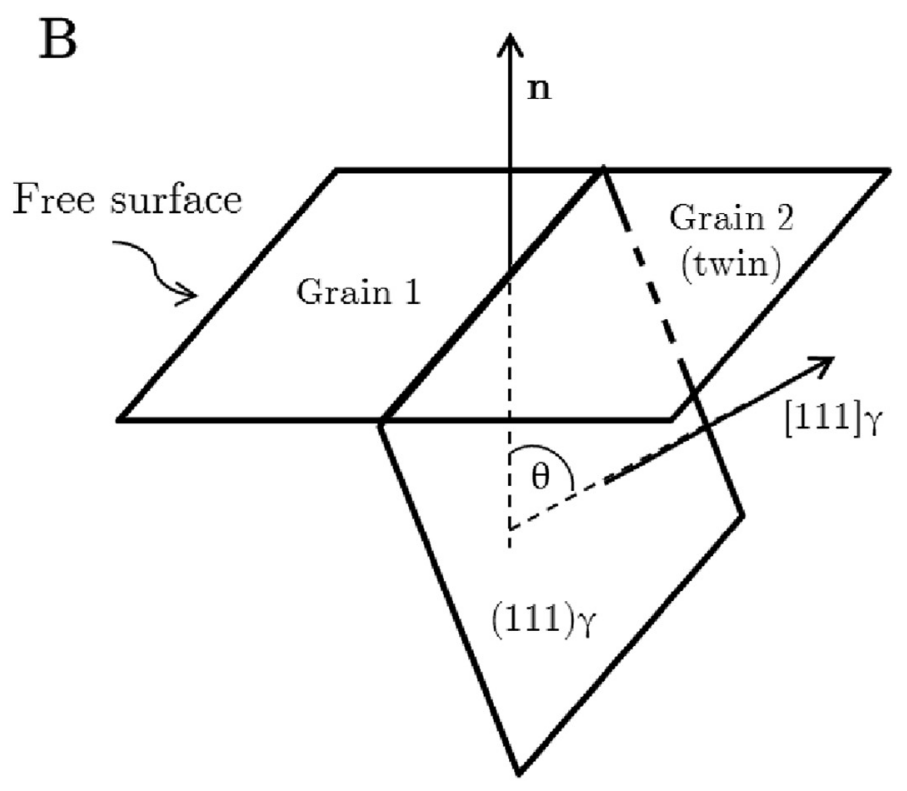

$\mathrm{D}$

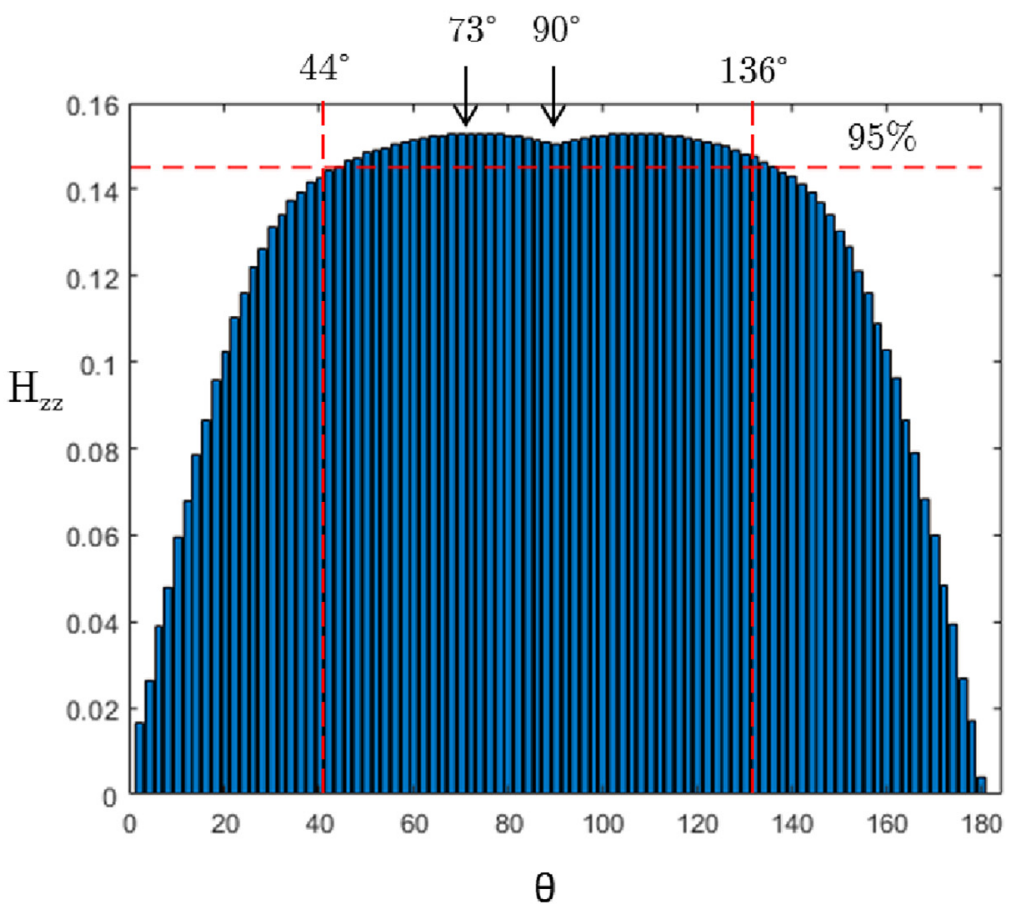

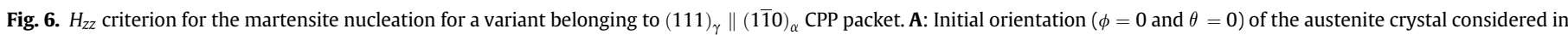

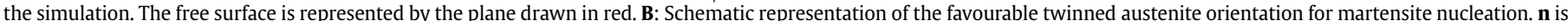

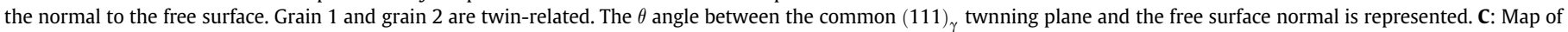

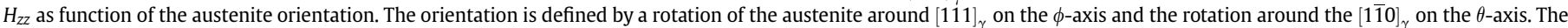

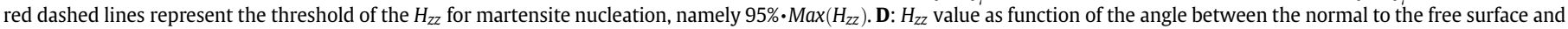

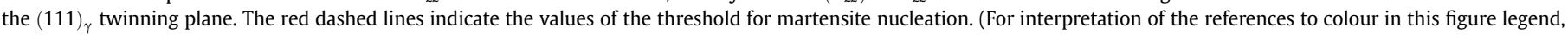
the reader is referred to the Web version of this article.)

performed for the six different variants that belong to the same CPP packet associated with the (111) $)_{\gamma}$ twin boundary. Fig. 7A-F shows the value $H_{z z}$ of all variants as function of the $\theta$ and $\phi$ angles of rotation around $[1 \overline{1} 0]_{\gamma_{1}}$ and the $[111]_{\gamma_{1}}$. The map in Fig. $7 \mathbf{A}$ represents the $H_{z z}$ of variant 1 in grain 2, and in Fig. 7B-F the maps of the $H_{z z}$ of the other 5 variants inside the CPP packet are illustrated. The pairs of maps (A-B), (C-D) and (E-F) in Fig. 7 are very similar. This similarity was expected as these are pairs of low-misoriented variants. In each Fig. $7 \mathbf{A}-\mathbf{F}$, the orientations satisfying the $95 \% \cdot \mathrm{H}_{z z}$ criterion in grain 2 are marked in white. We also report in black the orientations that favour the nucleation of variant 1 in grain 1 . They correspond to the orientations marked in white in Fig. $6 \mathbf{C}$.

The orientations that favour the nucleation of martensite variants on both side of the twin boundaries are the intersection of the 
A

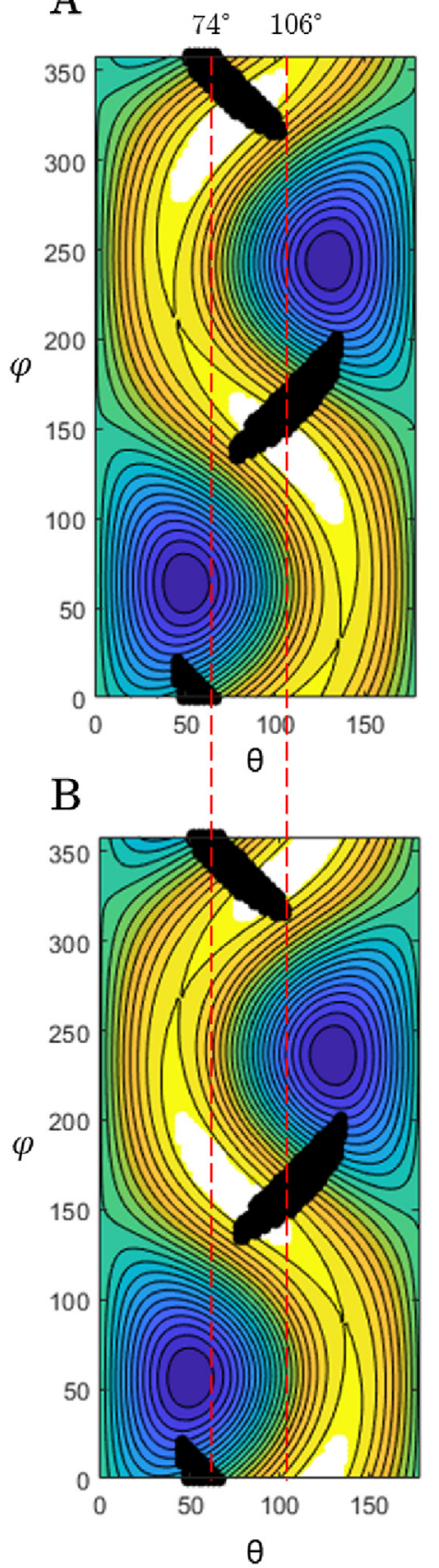

C

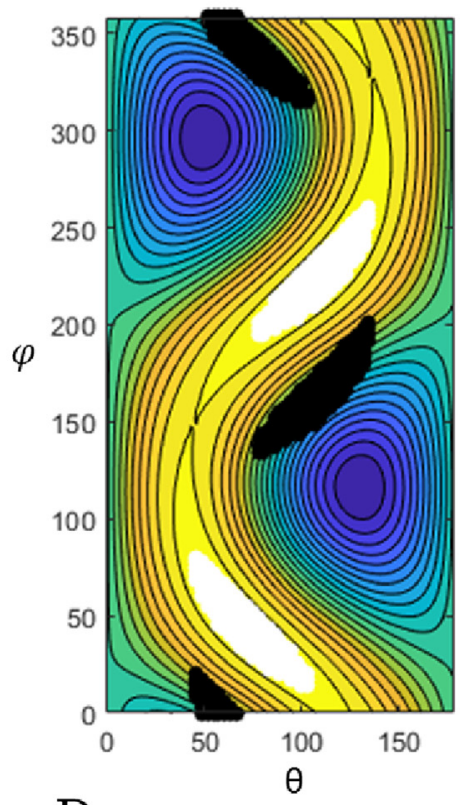

$\mathrm{D}$

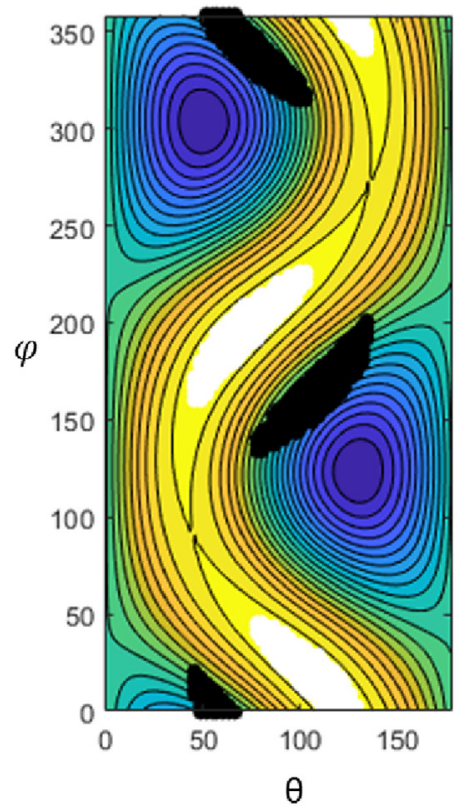

$\mathrm{E}$

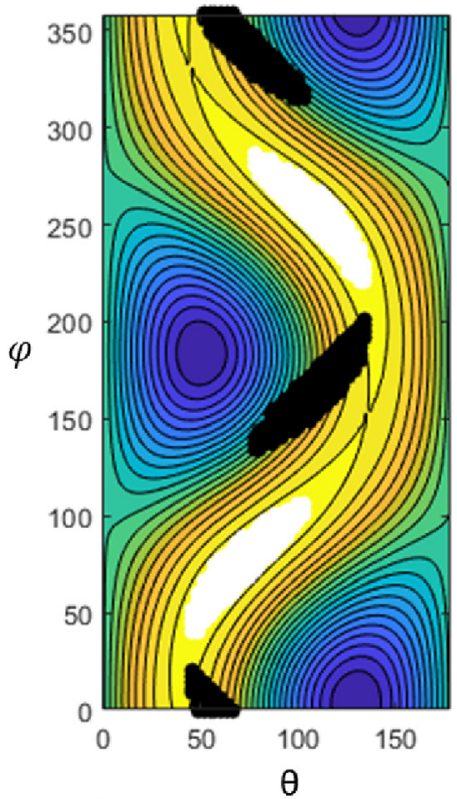

$\mathrm{F}$

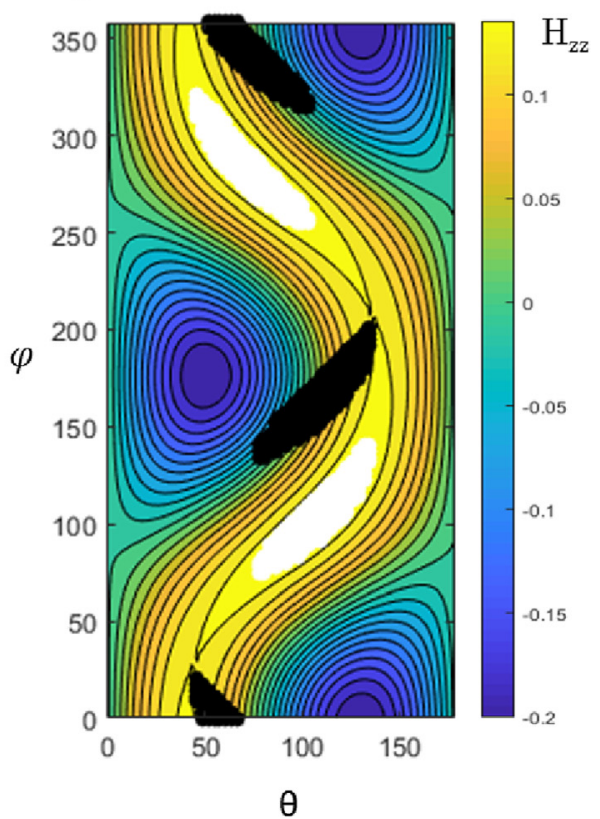

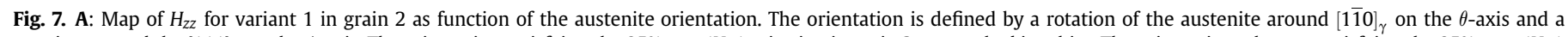

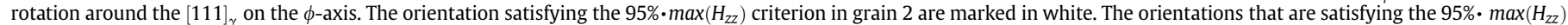

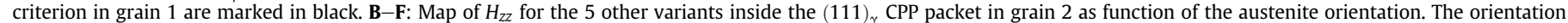

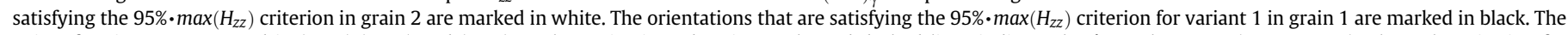

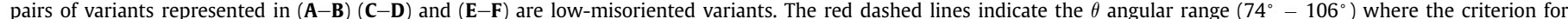

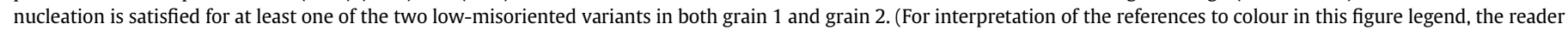
is referred to the Web version of this article.)

black and the white clouds. It appears that the clouds intersect only for variant 1 (Fig. 7A) and its low-misoriented variant (Fig. 7B). These results indicate that if a twin boundary is oriented such that the nucleation of a given variant is promoted on one side of the twin boundary (grain 1), the nucleation of martensite on the other side of the boundary (grain 2) is favoured only for the variants whose orientation is either the same or low-misoriented with the variant favoured in grain 1 . This result is in agreement with the experimental results in Fig. 2 showing that the martensite orientation spreads between two low-misoriented Kurdjumov-Sachs variants. The computation further indicates that the nucleation of martensite on both sides of the twin boundary is favoured when the twin boundary is exhibiting an angle above $74^{\circ}$ with respect to the free surface. The condition that the twinned austenite should satisfy for nucleation on both side of the twin boundary, resulting in the formation of the chevron, is thus more restrictive than the 
condition for the nucleation on only one side of the boundary, which is associated with the formation of half chevron $\left(44^{\circ}\right)$. Taken together, the results well agree with the experimental measurements. They indicate that for angles between $44^{\circ}$ and $74^{\circ}$, the martensite tends to form only in one side of the boundary, which experimentally corresponds to magenta bars in Fig. 4B, and full chevron (green bars) should be observed for angles greater than $74^{\circ}$. It can be noted however that some full chevrons are observed for angle between $55^{\circ}$ and $70^{\circ}$, which are not accounted by our calculations. For such orientations, we predict that the transformation will take place only in one side of the twin boundary. This discrepancy could indicate that the nucleation on one side of the boundary promotes the nucleation on the other side. In other words, if the criterion for nucleation is satisfied on one side (grain 1) the computed $H_{z z}$ value on the other side (grain 2) can be a little bit lower than the threshold value for nucleation and still the transformation occurs in both grains. It is also interesting to note that the thereshold value found in this study is larger than the one we used in our previous study [5]. In the latter case, to account for all the variants formed, the most appropriate threshold was set to 0 . Only the variants having negative value of $H_{z z}$ i.e., those associated with a contraction along the normal to the free surface were not formed. It seems thus that the initial state of the material affects the value of the threshold. Indeed, the sample used in Ref. [5] was an as-cast sample, whereas this one has been annealed after workhardening. It should be acknowledged also that in each case the threshold has been set to mimik the experimental data and, as such, it should be considered with care. In our previous work, we noted that using the variation $\dot{H}_{z z}$ of the $H_{z z}$ component at the beginning of the transformation seemed a good criterion for the variant selection. This criterion has been tested here and does not lead to more convincing results. We tested also whether the invariant plane strain (IPS) predicted by the PTMC was able to account for the variant selection observed in the present work. Our computations shows that given the austenite orientation the PTMC is able to predict fairly well the variants that form preferentially. However, this model cannot account for the particular orientations of the austenite that favours the martensite nucleation. The details of the computation are available in the supplement $\mathrm{C}$.

\section{Conclusions}

In conclusion, surface martensite has been formed in Fe-30 wt\% $\mathrm{Ni}$ recrystallized sample during electropolishing. The transformation occurs preferentially on twin boundaries, and the martensite formed on that boundaries exhibits a highly symetrical chevron morphology. EBSD measurements indicate that the transformation is favoured on twin boundaries that are rather vertical with respect to the sample surface, and that the martensite is in Kurdjumov-Sachs orientation relationship with both twinrelated austenite grains. The characterizations further show that the variants forming on the two sides of the twin boundary are the same or low-misoriented and that they belong to the CPP packet associated with the twin boundary. Among the six variants of the CPP packet, a maximum of two variants per twin boundray are observed indicating a variant selection phenomenon inside the CPP packet. The variant selection is adressed by using the criterion for variant selection proposed in our recent study on surface martensite based on the maximization of the extension in a direction normal to the free surface [5]. We show that starting from two experimental twin-related austenite orientations the criterion correctly predicts the martensite orientation formed on the twin boundary in three different EBSD maps. We then use the same criterion to explain why the rather vertical twin boundaries are preferred nucleation sites. Stating that the martensite nucleates on twin boundaries and that it belongs to the CPP packet associated with the twin boundary on which it nucleates, we have evaluated our criterion for surface martensite formation for all possible orientations of the austenite. The calculations were performed for each of the six variants composing the CPP packet. They indicate that only the austenite orientations associated with rather vertical twin boundaries maximize the criterion for nucleation on both side of the twin boundary, and that among the six variants, the favoured ones are either low-misoriented or the same in the two austenite grains. This study explains the selection of the twin boundaries promoting the transformation and hence rationalizes the highly symmetrical chevron morphology observed in surface martensite nucleating on twin boundaries.

\section{Acknowledgments}

We would like to thanks the Swiss National Science Foundation for supporting this research (grant number 200021-159955). The authors also acknowledge ArcelorMittal, in particular Kangying Zhu for providing the material of this study. Finally, we acknowledge PX Group for its financial support to the laboratory.

\section{Appendix A. Supplementary data}

Supplementary data to this article can be found online at https://doi.org/10.1016/j.actamat.2019.08.021.

\section{References}

[1] J. Klostermann, W. Burgers, Surface martensite in iron-nickel, Acta Metall. 12 (4) (1964) 355-360.

[2] J. Breedis, Martensitic transformation in iron-chromiun-nickel alloys, Trans. AIME 57 (230) (1964) 1583-1595.

[3] B. He, M. Huang, A. Ngan, S. Van Der Zwaag, Effect of free surface on the stability of individual retained austenite grains in a duplex stainless steel, Metall. Mater. Trans. A 45 (11) (2014) 4875-4881.

[4] K. Wakasa, C. Wayman, Crystallography and morphology of surface martensite in fe-20\% ni-5\% mn, Scr. Metall. 13 (12) (1979) 1163-1166.

[5] A.P. Baur, C. Cayron, R.E. Logé, Variant selection in surface martensite, J. Appl. Crystallogr. 50 (6) (2017) 1646-1652.

[6] M. Jaswon, J. Wheeler, Atomic displacements in the austenite-martensite transformation, Acta Crystallogr. 1 (4) (1948) 216-224.

[7] C. Cayron, Continuous atomic displacements and lattice distortion during fcc-bcc martensitic transformation, Acta Mater. 96 (2015) 189-202.

[8] S. Kajiwara, Roles of dislocations and grain boundaries in martensite nucleation, Metall. Mater. Trans. A 17 (10) (1986) 1693-1702.

[9] R. Yeo, Growth of martensite in an iron-28.8\% nickel alloy, Am. Soc. Met. Transp. Q. 57 (1964) 48-61.

[10] C. Magee, The nucleation of martensite, in: Phase Transformation, vol. 3, 1970, pp. $115-153$.

[11] Y. Sano, S. Chang, M. Meyers, S. Nemat-Nasser, Identification of stress-induced nucleation sites for martensite in fe-31.8 wt\% ni-0.02 wt\% c alloy, Acta Metall. Mater. 40 (2) (1992) 413-417.

[12] Z. Nishiyama, Martensitic Transformation, Elsevier, 2012.

[13] T. Tomida, M. Wakita, M. Yasuyama, S. Sugaya, Y. Tomota, S. Vogel, Memory effects of transformation textures in steel and its prediction by the double kurdjumov-sachs relation, Acta Mater. 61 (8) (2013) 2828-2839.

[14] F. Archie, S. Zaefferer, On variant selection at the prior austenite grain boundaries in lath martensite and relevant micro-mechanical implications, Mater. Sci. Eng., A 731 (2018) 539-550.

[15] T. Furuhara, T. Maki, Variant selection in heterogeneous nucleation on defects in diffusional phase transformation and precipitation, Mater. Sci. Eng., A 312 (1-2) (2001) 145-154.

[16] C. Cayron, EBSD imaging of orientation relationships and variant groupings in different martensitic alloys and widmanstätten iron meteorites, Mater. Char. 94 (2014) 93-110.

[17] D. Sarma, J. Whiteman, S. Keown, The structure of burst and isothermal martensites in an fe-24 wt\% ni-0.5 wt\% c alloy, J. Mater. Sci. 14 (3) (1979) 693-698.

[18] H. Sato, S. Zaefferer, A study on the formation mechanisms of butterfly-type martensite in fe-30\% ni alloy using ebsd-based orientation microscopy, Acta Mater. 57 (6) (2009) 1931-1937.

[19] M. Umemoto, T. Hyodo, T. Maeda, I. Tamura, Electron microscopy studies of butterfly martensite, Acta Metall. 32 (8) (1984) 1191-1203. 